Original Article

\title{
Analysis of the muscular activities of the tibialis anterior and gastrocnemius muscles in functional reach
}

\author{
SE-Won Yoon, PhD ${ }^{1)}$ \\ 1) Department of Physical Therapy, Kwangju Women's University: 165 Sanjeong-dong, Gwangsan-gu, \\ Gwangju 506-713, Republic of Korea
}

\begin{abstract}
Purpose] This study aimed to examine the changes in the muscle activities of each section of the tibialis anterior and gastrocnemius muscles in the functional reach test. [Subjects and Methods] This study selected those who can walk independently, can reach out their arm up to over $25 \mathrm{~cm}$, can show over $90^{\circ}$ shoulder joint bending, have not undergone an operation in the lower limb joint, and are not taking any medication affecting their ability to keep their balance from among 24 elderly males and 24 elderly females. The muscular activities of the tibialis anterior and gastrocnemius muscles were measured using the functional reach test and electromyogram. The functional reach test was conducted at a total of four sections: $0,15,20$, and $25 \mathrm{~cm}$. [Results] Gender and age affected the muscular activity by section in the functional reach test. It was also found that the gastrocnemius muscle was used more than the tibialis anterior muscle to keep the balance, and the aged subjects with good balance ability showed no great change in muscular activity on both stable and unstable ground. [Conclusion] It was found that the subjects used the tibialis anterior muscle more, and the lower limbs of the frequently used part, to keep their balance. As the aged subjects had good balance ability, they showed no great change in muscular activity on both stable and unstable ground.

Key words: Functional reach test, Electromyogram
\end{abstract}

(This article was submitted Oct. 27, 2016, and was accepted Feb. 13, 2017)

\section{INTRODUCTION}

Due to the increase of the elderly population, the social interest in the elderly is increasing ${ }^{1)}$. Of the elderly aged 65 years or older, $20-40 \%$ experience falls every year ${ }^{2}$. The fall of the elderly is usually regarded as a multi-factorial etiological problem, including intrinsic and extrinsic risk factors. Generally, intrinsic risk factors refer to the characteristics of the individual (e.g. dementia, visual impairment, neurological and musculoskeletal disabilities, and postural hypotension) ${ }^{3)}$. Functional reach is measured as the maximum distance a subject can reach forward beyond arm length at shoulder height while maintaining a fixed base of support in the standing position ${ }^{4}$. Duncan et al. ${ }^{5}$ reported that it has a particularly high association with clinical balance ability assessment in the elderly. The exercise strategies that are used to improve the functional reach include the ankle strategy, which utilizes the ankle muscles; the range-of-motion strategy mainly through the hip joint, a strategy that utilizes the rotation of the torso; and the squat strategy, which utilizes the curves of the ankles, knees, and hip joints $^{6}$. Young people mainly use the ankle strategy while the elderly use the hip joint strategy ${ }^{7)}$.

According to the study of Nam et al. ${ }^{8}$ results showed that both the balance-training and ankle joint exercise groups had a significant increase in muscle activity in the tibialis anterior and gastrocnemius. Kim and $\mathrm{Oh}^{9)}$ applied isokinetic exercise to strengthen the knee joint muscle, and conducted the functional reach test. As a result, they reported muscle strength and balance ability improvement. Therefore, the purpose of this study was to investigate the changes in the methods of physical 
Table 1. Comparison of the muscle activities of the tibialis anterior and gastrocnemius at functional reach

\begin{tabular}{llcccc}
\hline & & \multicolumn{3}{c}{ Functional reach section } \\
\cline { 3 - 6 } & & $0 \mathrm{~cm}$ & $15 \mathrm{~cm}$ & $20 \mathrm{~cm}$ & $25 \mathrm{~cm}$ \\
\hline \multirow{2}{*}{ Tibialis anterior } & Stable ground & $0.002 \pm 0.001$ & $0.003 \pm 0.003$ & $0.005 \pm 0.004$ & $0.006 \pm 0.004$ \\
& Unstable ground & $0.002 \pm 0.002$ & $0.004 \pm 0.003$ & $0.005 \pm 0.004$ & $0.006 \pm 0.006$ \\
\hline \multirow{2}{*}{ Gastrocnemius } & Stable ground & $0.004 \pm 0.002$ & $0.009 \pm 0.004$ & $0.012 \pm 0.006$ & $0.017 \pm 0.021$ \\
& Unstable ground & $0.004 \pm 0.003$ & $0.008 \pm 0.004$ & $0.011 \pm 0.005$ & $0.015 \pm 0.007$ \\
\hline
\end{tabular}

balance control ability by section through the measurement of the muscle activities of the tibialis anterior and gastrocnemius muscles at functional reach on both stable and unstable ground.

\section{SUBJECTS AND METHODS}

This study was performed by selecting a total of 48 elderly persons ( 24 males, 24 females) aged 60 or older. All subjects signed an informed consent form prior to participation. All the subjects signed an informed-consent form, and the study was approved by K Women's University.

To analyze the stability muscles of the lower limbs, MP-150 (Biopac, USA) was used, and by interlocking with a computer, the electromyogram (EMG) software was used. Sampling was done with 1,000 Hz, and the bandpass filter was set to 20-500 Hz. For the electrodes, TSD150B, a rectangular bipolar surface electrode, was used. To reduce the skin resistance to the surface EMG signals, after removing the hairs and keratins, the site was disinfected with medical alcohol cotton, and the electrodes were placed after the site was completely dried. The electrodes were attached to the active site, muskelbauch, parallel to the muscle direction, by maximally contracting in the position where the maximum resistance can be given to the tibialis anterior and gastrocnemius muscles. The activity of the lower-limb muscle was measured using the RMS (root mean square) value of the EMG in five sections, where 10 seconds was maintained after the surface electrodes were attached to the tibialis anterior and gastrocnemius muscles. To reduce the muscle activity error, the average of the values for 5 seconds (excluding the first 3 seconds and the last 2 seconds) of each section was analyzed.

The functional reach ability was measured using a mental ruler in which $0,15,20$, and $25 \mathrm{~cm}$ were marked. The starting point was marked by measuring the end of the third metacarpal bone when the subjects leaned on the wall, bent over shoulder joint by $90^{\circ}$, completely stretched out the elbow joint, and stretched the hands forward parallel to each other. The subjects performed arm stretch horizontal to the measurement instrument as much as possible for each 'cm', and maintained the said pose for 10 seconds at each section. For more than $25 \mathrm{~cm}$, the point where the subjects could maintain the position for 10 seconds was marked. Additionally, if a subject moved his/her foot, the point was remeasured. For the experiment on unstable ground, the subjects put their two feet on a cushioned mat (horizontal: $18 \mathrm{~cm}$; vertical: $35 \mathrm{~cm}$ ), and the experiment was conducted in the same way as before. The subjects did the exercise once, and between measurements, a 15 -second break was given to prevent muscle fatigue.

The measured data were statistically processed using SPSS version 12.0. For the general characteristics of the subjects, descriptive statistics were used, and for the comparison of the muscle activity of functional reach, repeated-measures ANOVA with two-way was performed. The significance level $(\alpha)$ was set to 0.05 for statistical testing.

\section{RESULTS}

There was a significant difference in the section at the main-effect test of the tibialis anterior muscle ( $<<0.05$ ), but no significant differences were shown in the interaction between the ground and the section and in the main effect of the ground (Table 1). There was a significant difference in the section at the main-effect test of the gastrocnemius muscle ( $p<0.05$ ), but no significant differences were shown in the interaction between the ground and the section and in the main effect of the ground (Table 1).

\section{DISCUSSION}

When the functional reach test was first applied, the purpose was to assess the dynamic balance of the elderly with weak balance $^{10)}$. Age-related alterations in lower extremity joint moment suggest there is a difference in the support strategy between the elderly and the young ${ }^{11)}$. Therefore, this study aimed to determine the difference in muscle activity between the tibialis anterior muscle and the gastrocnemius muscle in each section at functional reach that assessed the dynamic balance ability.

$\mathrm{Kim}^{12)}$ reported that in their study, the tibialis anterior and gastrocnemius muscles had higher muscle activities on unstable ground than on stable ground. Kim et al. ${ }^{13)}$ stated that the muscle activity on unstable ground of the hemiplegic patients in 
their study provided balance or stability during walking through lower-limb muscle activity to compensate for the instability. In this study, the muscle activities at functional reach on stable and unstable ground were compared, but there was no significant difference between the two, unlike in the aforementioned previous papers. Gribble et al. ${ }^{7}$ reported that the young people in their study mainly used the ankle joint strategy as a strategic element of balance control, but the elderly used the hip joint strategy. In this study, there was no statistically significant difference between stable and unstable ground, and when the above study results are taken together, the reason for this is that the elderly used the hip joint strategy to keep their balance on unstable ground.

Lee et al. ${ }^{14)}$ stated that the ankle joints and muscles were structured in their study to contribute to movement as well as stability as a terminal structure of the lower limb. Woo et al. ${ }^{15)}$ reported that when there was swaying in the front of the body in their study, to keep one's balance in stable standing, the gastrocnemius muscle was mainly activated, and when there was swaying in the back of the body, the tibialis anterior muscle acted much. As with the previous studies, this study showed that the gastrocnemius muscle was mainly activated while the center of the body gradually moved forward at functional reach. In particular, the muscle activity increased in both the tibialis anterior and gastrocnemius muscles as the section of functional reach became longer. It is thought that as the section of functional reach on unstable ground became longer, greater physical sway occurred, and consequently, the tibialis anterior and gastrocnemius muscles jointly contributed to keeping one's balance.

It is thought that the ankle joint strategy increases the ability to maintain one's balance as the postural instability increases according to the lengthening of the section at functional reach. The limitations of this study are as follows. First, the FRT value increases even only during trunk rotation at functional reach, but the trunk rotation was not controlled in this study. Second, this study was targeted at the elderly with a good ability to balance and who could perform functional reach up to more than $25 \mathrm{~cm}$, not at the elderly with a disability or with a reduced ability to balance. Therefore, further studies are required with different study subjects and using different implementation methods. Additionally, the data of the subjects were not normally distributed; thus, if further studies are to be conducted with the data fulfilling the normality assumption, different results may be obtained.

\section{REFERENCES}

1) Kim JW, Han SW, Kim JG: The effects of elastic resistance exercise of lumbo-pelvic region and lower limbs muscle on walking ability and balance ability of the elderly. J Phys Growth Mot Dev, 2006, 14: 13-26.

2) Stel VS, Smit JH, Pluijm SM, et al.: Balance and mobility performance as treatable risk factors for recurrent falling in older persons. J Clin Epidemiol, 2003, 56: 659-668. [Medline] [CrossRef]

3) Ikezoe T, Asakawa Y, Tsutou A: The relationship between quadriceps strength and balance to fall of elderly admitted to a nursing home. J Phys Ther Sci, 2003, 15: 75-79. [CrossRef]

4) Kage H, Okuda M, Nakamura I, et al.: Comparison of the one-arm and tow-arm functional reach test in young adults. J Phys Ther Sci, 2009, 21: 207-212. [CrossRef]

5) Duncan PW, Studenski S, Chandler J, et al.: Functional reach: predictive validity in a sample of elderly male veterans. J Gerontol, 1992, 47: M93-M98. [Medline] [CrossRef]

6) Park JS, Kwon OY, Choi HS, et al.: Effects of the movement strategies on functional forward reach in standing. Korean Res Soc Phys Ther, 2000, 7: 46-54.

7) Gribble PA, Hertel J: Effect of hip and ankle muscle fatigue on unipedal postural control. J Electromyogr Kinesiol, 2004, 14: 641-646. [Medline] [CrossRef]

8) Nam SM, Kim WB, Yun CK: Effects of balance training by knee joint motions on muscle activity in adult men with functional ankle instability. J Phys Ther Sci, 2016, 28: 1629-1632. [Medline] [CrossRef]

9) Kim TH, Oh DS: Effects of exercise training on strength and balance for the elderly. Korean Res Soc Phys Ther, 2000, 7: 32-37.

10) Duncan PW, Weiner DK, Chandler J, et al.: Functional reach: a new clinical measure of balance. J Gerontol, 1990, 45: M192-M197. [Medline] [CrossRef]

11) Toda H, Nagano A, Luo Z: Age and gender differences in the control of vertical ground reaction force by the hip, knee and ankle joints. J Phys Ther Sci, 2015, 27: 1833-1838. [Medline] [CrossRef]

12) Kim TY: Effect that get in ankle muscle by knee angle stability and the unstable floor. Korea Sport Research, 2006, 17: 263-274.

13) Kim EJ, Hwang-Bo G, Lee SY, et al.: The effects of gait component and muscular activity on unstable surface balance training in stroke patients. J Rehabil Res, 2010, 14: 329-346.

14) Lee JW, Yoon SW, Kim JH, et al.: The effect of ankle range of motion on balance performance of elderly people. J Phys Ther Sci, 2012, 24: 991-994. [CrossRef]

15) Woo YK, Park JW, Choi JD, et al.: Electromyographic activities of lower leg muscles during static balance control in normal adults. Phys Ther Korea, 2004, 11: $35-45$. 